PROCEEDINGS OF THE

AMERICAN MATHEMATICAL SOCIETY

Volume 30, No. 3, November 1971

\title{
AN EXCEPTIONAL SET FOR INNER FUNCTIONS
}

\author{
RENATE MCLAUGHLIN
}

Abstract. Suppose $f$ is an inner function that is not a constant and not a finite Blaschke product. Let $E(f)$ denote the set of values inside the unit disk that are not assumed infinitely of ten by $f$. We show that $E(f)$ is an $F_{\sigma}$-set.

Let $D$ denote the open unit disk. A function $f(z)$, defined and analytic in $D$, is called an inner function if $|f(z)| \leqq 1(z \in D)$ and if $\lim _{r \rightarrow 1}\left|f\left(r e^{i \theta}\right)\right|=1$ for almost all $\theta(0 \leqq \theta<2 \pi)$. Throughout this note, we assume that an inner function is neither a constant nor a finite Blaschke product.

With each inner function $f$, the exceptional set

$$
E(f)=\{w:|w|<1, f \text { assumes } w \text { at most finitely often }\}
$$

is associated. It is known that $E(f)$ has capacity zero [1, p. 35], and it is also known that for each closed subset $S$ of capacity zero in the open unit disk there exists an inner function that omits every value in $S$ and no other values [1, p. 37 ], [3].

We prove the following result.

Theorem. For each inner function $f$, the set $E(f)$ is an $F_{\sigma}$-set of capacityzero.

Suppose $f$ is analytic and nonconstant in $D$, and let $R(f)$ $=\{w: w=f(z), z \in D\}$. For $n=1,2, \cdots$, define

$E_{n}(f)=\{w: w \in R(f)$;

$f$ assumes $w$ at most $n$ times, counting multiplicities $\}.$

Lemma. For each $n, E_{n}(f)$ is a closed subset of $R(f)$.

Proof. We have to show that if $f(z)$ assumes the values $w_{1}, w_{2}, \cdots$ $\left(w_{k} \in R(f)\right)$ at most $n$ times and if $w_{k} \rightarrow w(w \in R(f))$, then $f(z)$ assumes $w$ at most $n$ times, counting multiplicities.

Suppose $w_{1}, w_{2}, \cdots$ belong to $E_{n}(f)$ and $w_{k} \rightarrow w(w \in R(f))$. Assume there are $n+1$ solutions of the equation $f(z)=w$, say $z_{1}, \cdots, z_{n+1}$

Received by the editors August 28, 1970.

AMS 1970 subject classifications. Primary 30A76; Secondary 30A08.

Key words and phrases. Inner function, values that an inner function assumes at most finitely often, $F_{\sigma}$-set, Rouché's theorem.

${ }^{1}$ This research was supported in part by the National Science Foundation.

Copyright @ 1971, American Mathematical Society 
$\left(z_{k} \in D\right)$. Then we can find a simple, closed, rectifiable, positivelyoriented curve $C$ in $D$ such that $z_{1}, \cdots, z_{n+1}$ lie in the interior of $C$ and such that no possible further solutions of $f(z)=w$ lie on $C$. Set

$$
\min _{z \in C}|f(z)-w|=\epsilon .
$$

It follows that $\epsilon>0$. Choose $w_{m}$ such that $\left|w-w_{m}\right|<\epsilon / 2$. There are at most $n$ solutions of the equation $f(z)=w_{m}$. If necessary, change $C$ to a curve $C^{*}$ such that none of these solutions lies on $C^{*}$. The curve $C^{*}$ can be chosen in such a way that $z_{1}, \cdots, z_{n+1}$ are in the interior of $C^{*}$ and $|f(z)-w|>\epsilon / 2\left(z \in C^{*}\right)$. Therefore

$$
|f(z)-w|>\left|w-w_{m}\right| \quad\left(z \in C^{*}\right) \text {. }
$$

If we write

$$
f(z)-w_{m}=(f(z)-w)+\left(w-w_{m}\right),
$$

all hypotheses of Rouché's theorem are satisfied [2, p. 254], and it follows that $f(z)-w_{m}$ and $f(z)-w$ have the same number of zeros inside $C^{*}$. This is a contradiction.

If $f$ is an inner function, we define

$$
E_{0}(f)=\{w:|w|<1, f \text { omits } w\} .
$$

The set $E_{0}(f)$ is closed, since the range of $f$ is open. The theorem now follows from the fact that $E(f)=\bigcup_{n=0}^{\infty} E_{n}(f)$.

It is tempting to suspect that the sets

$$
E_{n}^{*}(f)=\{w: w \in R(f), f \text { assumes } w \text { exactly } n \text { times }\}
$$

are closed. This is false. It is not too difficult to think of the Riemann surface of a function that assumes $w=0$ exactly once and for which $w=0$ is the limit of both a sequence of points $w_{11}, w_{12}, \cdots$ that are assumed exactly once and a sequence of points $w_{21}, w_{22}, \cdots$ that are assumed exactly twice.

Finally, we remark that there exist inner functions for which $E_{0}(f) \neq \varnothing$ and each set $E_{n}(f)$ properly contains $E_{n-1}(f)$.

\section{REFERENCES}

1. E. F. Collingwood and A. J. Lohwater, The theory of cluster sets, Cambridge Univ. Press, Cambridge, 1966. MR 38 \#325.

2. E. Hille, Analytic function theory. Vol. 1, Introduction to Higher Math., Ginn, Boston, Mass., 1959. MR 21 \#6415.

3. G. Hössjer and O. Frostman, Über die Ausnahmestellen eines Blaschkeproduktes, Kungl. Fysiogr. Sällsk. Lund Förh. 3 (1933), no. 16, 8 pp.

University of Michigan-Flint, Flint, Michigan 48503 